\title{
Transport and hydrodynamic calculations of direct photons at FAIR
}

\author{
Bjørn Bäuchle ${ }^{1,2, \text { * }}$ and Marcus Bleicher ${ }^{1,2}$ \\ ${ }^{1}$ Frankfurt Institute for Advanced Studies, Frankfurt am Main, Germany \\ ${ }^{2}$ Institut für Theoretische Physik, Goethe-Universität, Frankfurt am Main, Germany
}

\begin{abstract}
The microscopic transport model UrQMD and a micro+macro hybrid model are used to calculate direct photon spectra from $\mathrm{U}+\mathrm{U}$-collisions at $E_{\mathrm{lab}}=35 \mathrm{AGeV}$ as will be measured by the CBM-collaboration at FAIR. In the hybrid model, the intermediate high-density part of the nuclear interaction is described with ideal 3+1-dimensional hydrodynamics. Different equations of state of the matter created in the heavy-ion collisions are investigated and the resulting spectra of direct photons are predicted. The emission patterns of direct photons in space and time are discussed.
\end{abstract}

\section{INTRODUCTION}

In order to understand the phases of compact stellar objects and phase transitions in the early universe, a deep understanding of the phase diagram of strongly interacting matter is needed. The experimental tool to gain these informations is heavy-ion physics, where large nuclei are collided. In those collisions, nuclear matter is heated to temperatures that are believed to be sufficient to cause a phase transition from a hadron gas to a state where partonic degrees of freedom are relevant (the Quark-Gluon-Plasma QGP). Also at lower energies, but very high baryon densities novel phenomena like color super conducting phases [1] or even Quarkyonic Matter [2] are expected in contrast to the well known hadron gas.

From lattice gauge theory calculations, it is deduced that the transition between the hadronic and QGP phase is a cross-over [3, 4], if the baryochemical potential $\mu_{\mathrm{B}}$ is sufficiently small. The transition temperature is expected to be around $T_{C} \approx 170 \mathrm{MeV}$ [5]. At high $\mu_{\mathrm{B}}$, first principle calculations of the phase structure are no longer possible, but symmetry arguments suggest that the phase transition line ends at high $\mu_{\mathrm{B}}$ and zero temperature $T=0$ as a first order phase transition. If so, then a critical end point must exist, at which the crossover turns into a first order phase transition.

The search for this critical end point is the main motivation for current and planned experimental heavyion programs of the SHINE-experiment at the Super Proton Synchrotron (CERN-SPS) [6] and the CBMexperiment at the Facility for Antiproton and Ion Reserach (FAIR) 7]. The current low-energy run at the Relativistic Heavy Ion Collider (BNL-RHIC) also addresses this issue [8, [9].

The CBM-collaboration will measure uraniumuranium collisions in a fixed-target experiment at incident energies of up to $E_{\text {lab }}=35 \mathrm{AGeV}$. The proposed features for this detector include, besides subsystems to measure hadronic observables, electromagnetic calorimetry in order to measure photons.

Electromagnetic probes such as leptons and photons

*Electronic address: baeuchle@th.physik.uni-frankfurt.de have the advantage that they do not suffer from final state interactions and hence carry all information from their production vertex into the detector [10]. Therefore, they also carry information from all stages of the heavyion collision. Hadronic probes, on the other hand, are mostly produced very late in the collision or rescatter in the late stages, so that they carry only indirect information from the early stages, such as flow patterns and fluctuations.

Previous calculations of direct photons from transport theory include work with UrQMD by Dumitru et al. 11] and Bäuchle et al. [12] and with HSD by Bratkovskaya et al. [13]. Hydrodynamics has been used in many direct photon calculations, see e.g. 14 21].

Most photons produced in heavy-ion collisions, however, come from hadronic decays, predominantly the decay of the $\pi^{0}$ and $\eta$. Due to the long lifetime of both particles, they decay far outside the collision zone. In order to extract information about the fireball from photons, the decay photon contribution has to be subtracted. The remaining photons, those that do not come from decays, are called direct photons. Experimental techniques for the extraction of direct photon yields from the inclusive measurements include the direct estimation of the background via invariant mass-analysis of the photons [22, 23], the analysis of interference patterns (using a Hanburry Brown-Twiss analysis) 24] and the extrapolation of the spectra of massive virtual photons (low-mass dileptons) to massless photons [25].

In this work, we investigate the influence of different evolutions of the bulk medium on the direct photon production in U+U-collisions at $E_{\mathrm{Lab}}=35 \mathrm{AGeV}$ using the model established in 12]. We compare calculations with hadronic degrees of freedom to calculations with a first order phase transition to a QGP and calculations with a cross-over to chirally restored and deconfined matter. In Section II we briefly present the model and parameters used for these calculations, and in Section III we show and analyse our calculations for direct photon emission.

\section{THE MODEL}

The microscopic transport model Ultrarelativistic Quantum Molecular Dynamics (UrQMD) [26 28] pro- 
vides the setting in which the present work is calculated. Calculations with UrQMD using standard options provides a baseline calculation with hadronic and string degrees of freedom throughout the nucleusnucleus-interaction and with vacuum properties of all particles.

Uranium has an eccentricity of $\epsilon=0.27$. However, for the present study, the uranium nuclei are initialized spherically, which provides an intrinsic averaging over different possible alignments of the projectile and target nucleus. We have checked that for untriggered collisions (i.e. without selection on the alignment of the nuclei) the results are not sensitive to this choice.

The hybrid option which is new in version 3.3 [29 [31, 36] allows to substitute the high-density part of a heavy-ion collision with an ideal 3+1-dimensional hydrodynamic calculation, where different assumptions on the matter present in the collision can be tested by varying the Equation of State. Non-equilibrium initial state interactions and final state scatterings in the dilute hadronic medium, as well as decays are calculated within the microscopic transport model.

The transition from the particle-based transport description to the density-based hydrodynamic description happens when the initial baryon-currents have decoupled. We estimate this to be when the initial nuclei have completely passed through each other, which in the case of the system investigated here $(\mathrm{U}+\mathrm{U}$-collisions at $\left.E_{\text {lab }}=35 \mathrm{AGeV}\right)$ is at $t_{\text {start }}=3.7 \mathrm{fm}$ after the very first scatterings. At this point, all particles that have interacted or are newly produced are used to calculate energy-density, baryon number-density and momentumdensities, which are then taken as input for the hydrodynamic calculation. During this transition, the system is forced into local thermal equilibrium, regardless of the state of actual equilibration of the matter.

After the hydrodynamic evolution has proceeded, the Cooper-Frye-formalism [32] is used to couple the hydrodynamic calculation to the late transport stage. This transition happens individually for each transverse slice, characterized by the same position along the beam direction, when all cells in that slice have diluted to below a critical energy density, whose exact value depends on the Equation of State employed (see Table 【). The hypersurface on which the transition happens is piecewise isocronous and non-continuous. However, since after that transition scatterings and decays are calculated with UrQMD, the effects of that should be negligible. For more details on the mappings, the reader is referred to Petersen et al. [31, 33].

\section{A. Equations of State}

Four different calculations are compared in the current work. Firstly, we compare direct photon spectra from hadronic systems, calculated in pure cascade model and in the cascade+hydrodynamic hybrid model with

\begin{tabular}{|c|c|}
\hline EoS & $\epsilon_{\text {crit }}$ \\
\hline HG-EoS & $5 \epsilon_{0}$ \\
\hline$\chi-\operatorname{EoS}$ & \\
\hline BM-EoS $\mid$ & \\
\hline
\end{tabular}

TABLE I: The critical energy densities for the mapping from hydrodynamics to transport theory for the various Equations of state. $\epsilon_{0}=146 \mathrm{MeV} / \mathrm{fm}^{3}$ is the nuclear ground state energy density.

Hadron-Gas EoS (HG-EoS) 34]. The HG-EoS has the same degrees of freedom as the transport phase and therefore provides excellent means to investigate the effects of instant thermalization at the transition from transport to hydrodynamic description and to investigate the effects of the different kinetic descriptions. The hybrid model is also used with a Chiral EoS $(\chi$ EoS) [35] featuring a rapid cross-over to chirally restored and deconfined matter, which is compared to the aforementioned hadronic descriptions and calculations with a MIT-Bag Model EoS (BM-EoS) with a strong firstorder phase transition. Both $\chi$-EoS and BM-EoS have their phase transition at vanishing chemical potential at around $T_{\mathrm{C}} \approx 170 \mathrm{MeV}$.

\section{B. Intermediate stage in cascade calculations}

From the considerations above, it is clear that the hybrid model consists of three stages, the pre-equilibrium early stage, the hydrodynamic intermediate stage and the dilute late stage. When comparing the stages between the different variations of the model and among each other, we assign the same division of stages to the pure cascade calculations. Along these lines we define the early stage as $0<t<3.7 \mathrm{fm}$, the intermediate stage as $3.7<t<11 \mathrm{fm}$ and the late stage after $t=11 \mathrm{fm}$.

\section{Photon emission sources}

In the present work, direct photon emission is treated as a perturbation on top of the evolution of the fireball, so the underlying evolution of the hadronic medium remains unaltered by the calculation of direct photon spectra. This is justified, because the ratio of electromagnetic cross-sections (producing the photons) and strong crosssections (governing the evolution of the bulk) is very small.

The most important hadronic channels for the production of direct photons are $\pi \pi \rightarrow \gamma \rho$ and $\pi \rho \rightarrow \gamma \pi$, which both are implemented in the transport, as well as in the hydrodynamic phase. The cross-sections for cascade-calculations are taken from Kapusta et al. [14], while the rates used for the hydrodynamic description have been parametrized by Turbide et al. [16]. Since no thermal partonic interactions are modelled in UrQMD, 


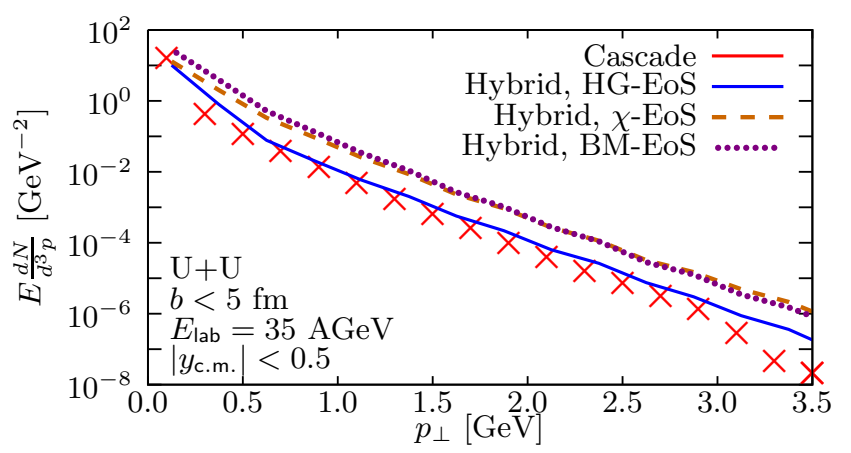

FIG. 1: (Color Online) Overall direct photon spectra from $\mathrm{U}+\mathrm{U}$-collisions. Calculations in pure cascade mode are shown as red crosses, hybrid calculations with HG-EoS (see text) are shown as solid blue line, $\chi$-EoS-hybrid calculations as orange dashed line and BM-EoS-hybrid calculations are shown as purple dotted line.

emission from a QGP-medium is only taken into account in the hydrodynamic part of the model. Several minor hadronic channels are only implemented in one of the two models, such as strange channels (e.g. $K \pi \rightarrow \gamma K^{*}$ ) which are only present in the hydrodynamic calculations, and $\eta$-channels (e.g. $\pi \eta \rightarrow \gamma \pi$ ) which are only present in the transport calculations. Earlier investigations with this model have shown those channels to contribute about equally, but not significantly, to the overall spectrum of direct photons. The complete list of channels and a detailed explanation of the calculation is provided in [12].

\section{RESULTS}

We start with a comparison of the overall direct photon spectra calculated with the various Equations of State and with the transport-only approach. From Figure 1 . one can clearly see that the transport-only (crosses) and HG-EoS (solid line) calculations give very similar results. The BM-EoS and $\chi$-EoS calculations, on the other hand, yield significantly higher spectra than both hadronic calculations. The reason for this difference will be discussed below.

Next, we investigate the contributions of the early, intermediate and late stages to the overall spectra for all variations of the model. In Figure2, we show that the intermediate stage, which is calculated with hydrodynamics in the hybrid calculations, dominates the emission in all cases, although the excess of this stage is less significant in the hadronic calculations of pure transport and HG-EoS hybrid calculations.

Let us now take a look at the intermediate stages and investigate the contribution of hadronic and partonic direct photon emissions to the spectra from $\chi$-EoS and BMEoS hybrid calculations. In Figure 3, we show the total contributions of the intermediate stages and the part of it that comes from hadronic sources (in case of the $\chi$ and BM-EoS-calculations). We note that the hadronic

\begin{tabular}{l|l|r||l} 
Calculation & $T_{\text {slope }}[\mathrm{MeV}]$ & $A\left[\mathrm{GeV}^{-2}\right]$ & $\chi^{2} /$ d.o.f. \\
\hline Transport & $198.0 \pm 6.6$ & $2.09 \pm 0.74$ & 0.559 \\
HG-EoS & $203.5 \pm 8.0$ & $2.98 \pm 1.16$ & 0.532 \\
$\chi$-EoS & $214.8 \pm 6.1$ & $7.57 \pm 2.02$ & 0.249 \\
BM-EoS & $200.8 \pm 5.8$ & $15.39 \pm 4.45$ & 0.291
\end{tabular}

TABLE II: Results of exponential fits to the spectra in the range $0<p_{\perp}<3.5 \mathrm{GeV}$. The fit function is $f\left(p_{\perp}\right)=$ $A \exp \left(-\frac{p_{\perp}}{T_{\text {slope }}}\right)$.

contributions are very similar in all cases, and that the excess observed in calculations with partonic degrees of freedom comes from the partonic part of the system. Figure 4 shows the relative contribution of the QGP-phase in hybrid calculations with BM- and $\chi$-EoS. The contribution remains fairly constant at transverse momenta $p_{\perp}>0.5 \mathrm{GeV}$, and is at about $85 \%$ for the $\chi$-EoS and at about $65 \%$ for the BM-EoS-calculations.

What is eye-catching with the spectra is that despite the obvious differences in magnitude, the slopes of the spectra are very similar. Indeed, a closer anaylsis reveals all inverse slope parameters to be about $T_{\text {slope }}=$ $200 \mathrm{MeV}$, with only the $\chi$-EoS calculations being slightly higher (see Table 【).

Since emission of direct photons happens throughout the heavy-ion collision, it is prudent to look at the emission times of photons. We do so in Figure 5 showing the average emission time of direct photons plotted against their transverse momentum for the two most abundant channels in cascade-only calculations and the time evolution of direct photon emission for various transverse momentum bins.

We can see that the average emission time is nearly constant at about $\langle t\rangle \approx 7.6 \mathrm{fm}$ over the whole $p_{\perp}$-range. It is interesting to note that at intermediate $p_{\perp}$ direct photons from $\pi \pi$-scatterings have a significantly lower average emission time $\left\langle t_{\pi \pi}\right\rangle \approx 4 \mathrm{fm}$ than those coming from $\pi \rho$-collisions.

The time evolution of direct photon emission in the lower part of Figure [5] shows that at intermediate transverse momentum $1.5<p_{\perp}<2.5 \mathrm{GeV}$ the emission has two peaks, one after roughly $3 \mathrm{fm}$ and the other one at $8 \mathrm{fm}$. The average emission time in this momentum region is $\left\langle t\left(p_{\perp} \approx 2 \mathrm{GeV}\right)\right\rangle=7.6 \mathrm{fm}$. At high transverse momentum, $2.5<p_{\perp}<3.5 \mathrm{GeV}$, no significant peak at $t>5 \mathrm{fm}$ is present, but the tail of the distribution is still large enough to have the average emission time as high as $\left\langle t\left(p_{\perp} \approx 3 \mathrm{GeV}\right)\right\rangle=6.4 \mathrm{fm}$.

At very low $p_{\perp}<0.5 \mathrm{GeV}$, the emission is dominated by $\pi \pi$-scatterings, and is symmetrically centered around its average value of $\left\langle t\left(p_{\perp} \approx 0 \mathrm{GeV}\right)\right\rangle=8.8 \mathrm{fm}$, as is the $\pi \rho$-dominated photon emission at low transverse momentum $0.5<p_{\perp}<1.5 \mathrm{GeV}$, whose average time is $\left\langle t\left(p_{\perp} \approx 1 \mathrm{GeV}\right)\right\rangle=7.6 \mathrm{fm}$.

When looking at the relative contributions of the different emission times $t_{\mathrm{em}}$ to the direct photon yield as a 


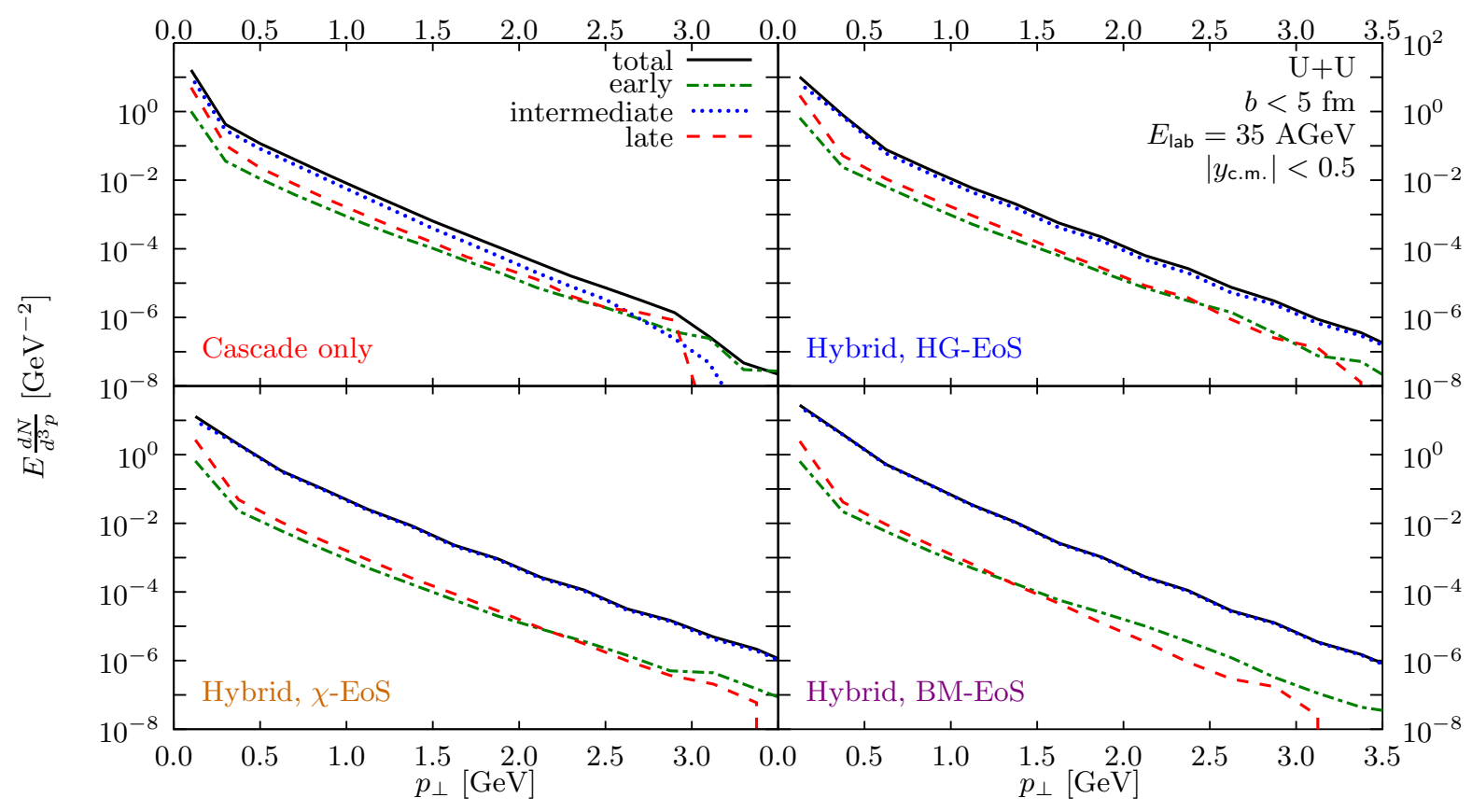

FIG. 2: (Color Online) The contributions of the early (green dash-dotted lines), intermediate (blue dotted lines) and late (red dashed lines) stages to the overall direct photon spectra (solid black lines) separately for all four variations of the model. Calculations in transport mode are shown in the top left panel, hybrid calculations with HG-EoS on the top right, $\chi$-EoS hybrid calculations are in the bottom left, and BM-EoS hybrid calculations are in the bottom right panel.

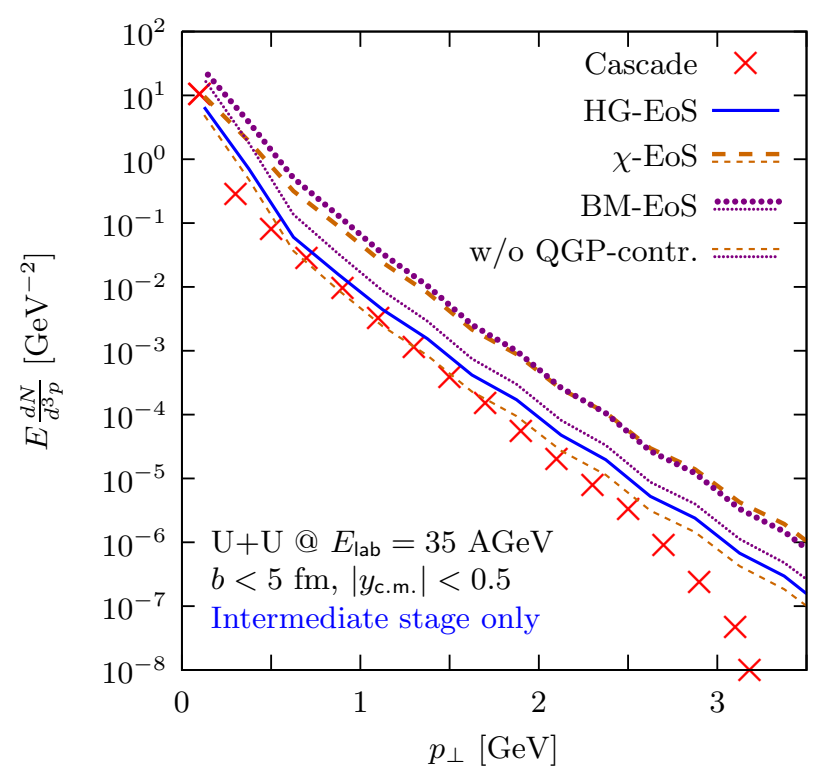

FIG. 3: (Color Online) Comparison of the contributions of the intermediate stages for all variations of the model. In addition, the hadronic contributions to the intermediate-stage emissions in $\chi$-Eos and BM-EoS are shown in thin lines.

function of direct photon transverse momentum in Figure 6. we can assert the picture presented above. At low transverse momenta $p_{\perp}<2 \mathrm{GeV}$, emission from $5<t_{\mathrm{em}}<10 \mathrm{fm}$ dominates and constitutes about $50 \%$ of

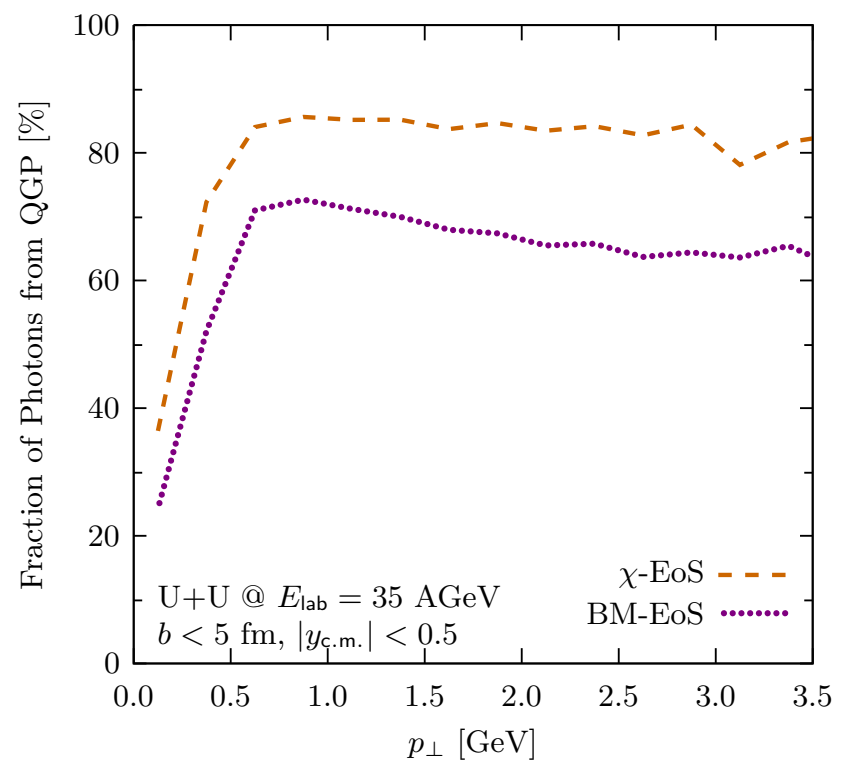

FIG. 4: (Color Online) The fraction of direct photons emitted from the QGP-phase in hybrid calculations with $\chi$-EoS (dashed orange line) and BM-EoS (purple dotted line) as a function of transverse photon momentum.

the overall contribution. At higher $p_{\perp}>2.5 \mathrm{GeV}$, however, emission from the very early stages of the collision starts to dominate. The contribution of photons emitted earlier than $t_{\mathrm{em}}=5 \mathrm{fm}$ contribute more than $60 \%$ to the 

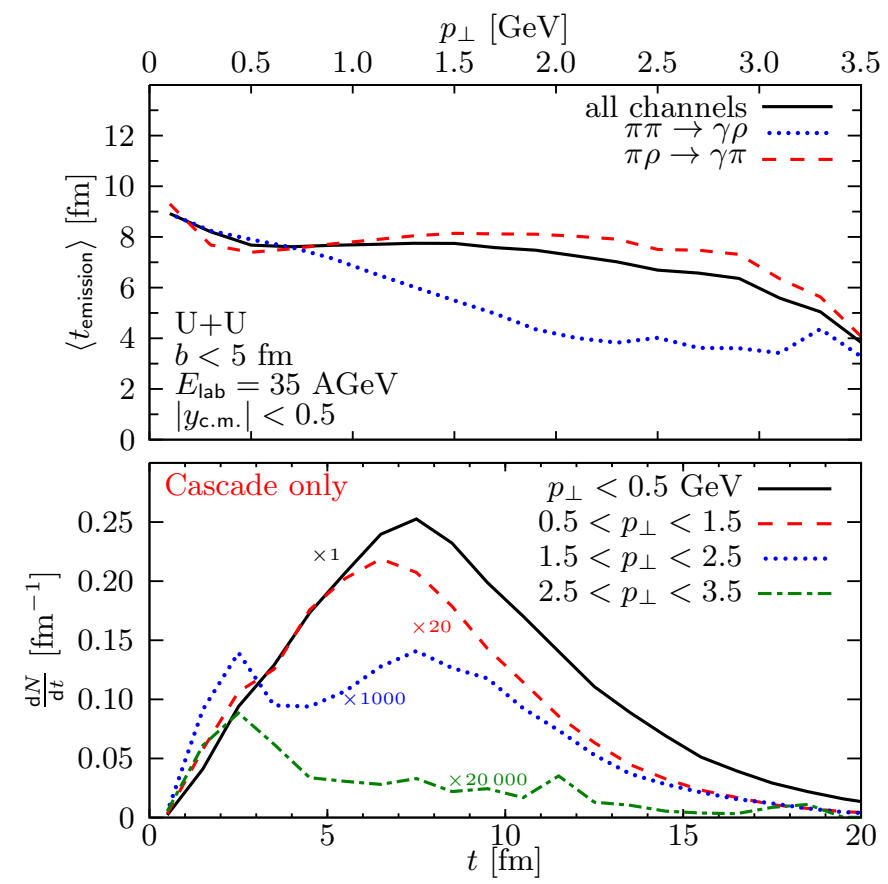

FIG. 5: (Color Online) Average emission time of direct photons in cascade calculations as function of $p_{\perp}$ (top) and time evolution of the photon emission for various transverse momenta (bottom). The time evolution patterns from high transverse momenta have been scaled for readability.

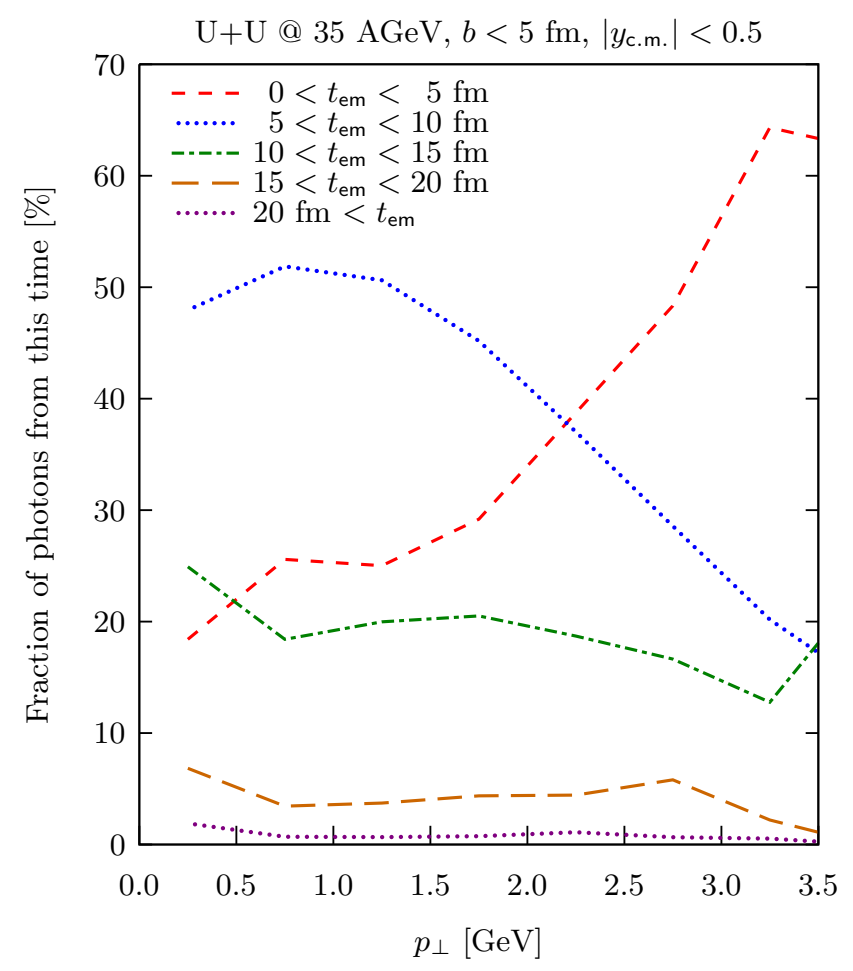

FIG. 6: (Color Online) Fraction of photons that are emitted from $0<t<5 \mathrm{fm}$ (red dashed line), $5<t<10 \mathrm{fm}$ (blue dotted line), $10<t<15 \mathrm{fm}$ (green dash-dotted line), $15<$ $t<20 \mathrm{fm}$ and $t>20 \mathrm{fm}$ (violett dotted line) as a function of transverse momentum.

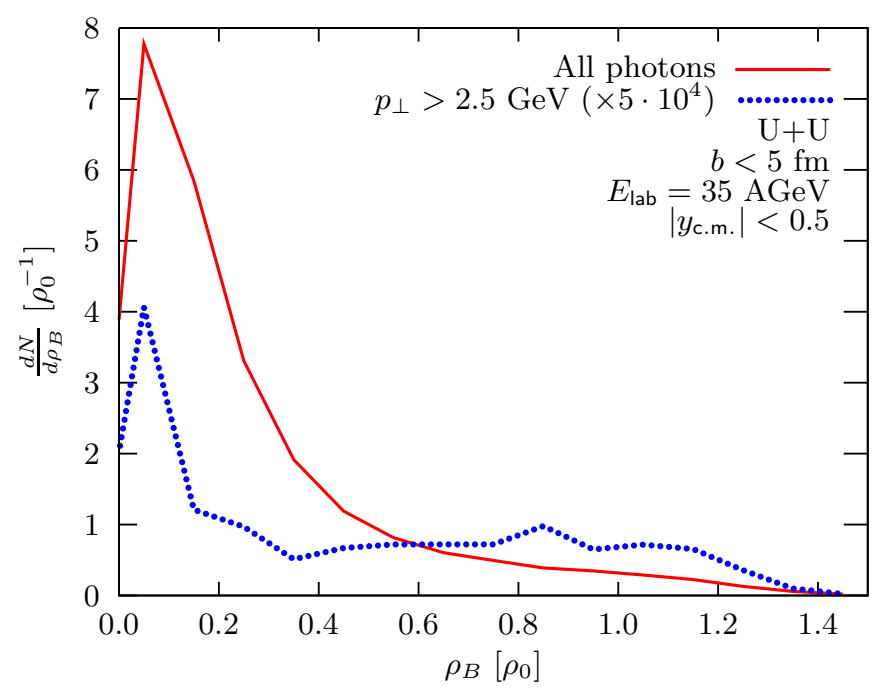

FIG. 7: Distribution of direct photons against the baryon number density $\rho_{\mathrm{B}}$ at the emission point for cascade calculations for all photons (red solid line) and for photons with $p_{\perp}>2.5 \mathrm{GeV}$ (blue dotted line, scaled by $5 \cdot 10^{4}$ ).

direct photon spectrum at $p_{\perp}>3 \mathrm{GeV}$.

The baryon number density at the point of emission of direct photons is investigated in Figure 7 . Most photons come from collisions below $\rho_{B}<0.5 \rho_{0}$, with $\rho_{0}=0.16 \mathrm{fm}^{-3}$ being the nuclear ground state baryon number density. At high transverse momenta, the photon emission is shifted from the low-density region towards high densities. We therefore assume it to be safe to employ vacuum cross-sections and vacuum thermal rates which do not take into account high-density effects on the $\rho$-spectral function.

\section{SUMMARY}

Direct photon emission from U+U-collisions at $E_{\mathrm{lab}}=$ $35 \mathrm{AGeV}$ with $b<5 \mathrm{fm}$ has been calculated and analysed within a microscopic transport model and in a micro+macro hybrid model, in which the high-density phase of the transport model has been replaced by ideal 3+1-dimensional hydrodynamic calculations. In the hybrid framework, three different Equations of State governing the behaviour of the matter in the hydrodynamic model have been compared.

We find that the presence of partonic degrees of freedom significantly enhance the amount of direct photons emitted from the medium, but not so much the slope of the direct photon spectra. At the energy under investigation, we find that this enhancement comes directly from the partonic phase and is not due to a prolonged hadronic lifetime or hadronic contributions from the mixed phase, which is present in the BM-EoS hybrid calculations.

The average emission time of direct photons is nearly constant at $\langle t\rangle \approx 7.6 \mathrm{fm}$ over a broad range of transverse momenta in the case of cascade-only calculations, and 
also the spectra from hybrid model calculations are dominated by emission from the intermediate stages. The average emission time of photons originating from $\pi \pi$ scatterings drop to lower times at transverse momenta larger than $p_{\perp}>1.5 \mathrm{GeV}$, but since at this $p_{\perp}$-range this process is subleading to the dominant $\pi \rho$-channel, this does not affect the overall average emission time. Emission is found to come dominantly from areas with low baryon number density. However, a trigger on high $p_{\perp}$ will allow to select photons from the early stage.

\section{OUTLOOK}

The predictions for FAIR-energies shown in this work lay the foundations for future work analysing the ratio between direct photon emission and decay photons. Further work with this model will include the calculation of direct photon spectra at RHIC-energy and an analysis of the effect of changing the interface-parameters between the transport and hydrodynamic models on the direct photon emission.

\section{ACKNOWLEDGEMENTS}

This work has been supported by the Frankfurt Center for Scientific Computing (CSC), the GSI and the BMBF. B. Bäuchle gratefully acknowledges support from the Deutsche Telekom Stiftung, the Helmholtz Research School on Quark Matter Studies and the Helmholtz Graduate School for Hadron and Ion Research. This work was supported by the Hessian LOEWE initiative through the Helmholtz International Center for FAIR.

The authors thank Elvira Santini for valuable discussions as well as Lars Zeidlewicz and Tim Schuster.
[1] D. H. Rischke, Prog. Part. Nucl. Phys. 52 (2004) 197 [arXiv:nucl-th/0305030].

[2] L. McLerran and R. D. Pisarski, Nucl. Phys. A 796 (2007) 83 [arXiv:0706.2191 [hep-ph]].

[3] Y. Aoki, G. Endrodi, Z. Fodor, S. D. Katz and K. K. Szabo, Nature 443 (2006) 675 [arXiv:hep-lat/0611014].

[4] P. de Forcrand and O. Philipsen, PoS LATTICE2008 (2008) 208 [arXiv:0811.3858 [hep-lat]].

[5] S. Borsanyi, Z. Fodor, C. Hoelbling, S. D. Katz, S. Krieg, C. Ratti and K. K. Szabo [Wuppertal-Budapest Collaboration], arXiv:1005.3508 [hep-lat].

[6] A. Laszlo et al. [NA61 Collaboration], PoS C POD07 (2007) 054 [arXiv:0709.1867 [nucl-ex]].

[7] C. Hohne [CBM Collaboration], Nucl. Phys. A $\mathbf{7 4 9}$ (2005) 141.

[8] H. Caines [STAR Collaboration], arXiv:0906.0305 [nuclex].

[9] M. M. Aggarwal et al. [STAR Collaboration], arXiv:1007.2613 [nucl-ex].

[10] C. Gale, arXiv:0904.2184 [hep-ph].

[11] A. Dumitru, M. Bleicher, S. A. Bass, C. Spieles, L. Neise, H. Stoecker and W. Greiner, Phys. Rev. C 57 (1998) 3271 [arXiv:hep-ph/9709487].

[12] B. Baeuchle and M. Bleicher, Phys. Rev. C 81 (2010) 044904 [arXiv:0905.4678 [hep-ph]].

[13] E. L. Bratkovskaya, S. M. Kiselev and G. B. Sharkov, Phys. Rev. C 78 (2008) 034905 [arXiv:0806.3465 [nuclth]].

[14] J. I. Kapusta, P. Lichard and D. Seibert, Phys. Rev. D 44 (1991) 2774 [Erratum-ibid. D 47 (1993) 4171].

[15] D. K. Srivastava and B. Sinha, Phys. Rev. C 64 (2001) 034902 [arXiv:nucl-th/0006018].

[16] S. Turbide, R. Rapp and C. Gale, Phys. Rev. C 69 (2004) 014903 [arXiv:hep-ph/0308085].

[17] S. Turbide, C. Gale, S. Jeon and G. D. Moore, Phys. Rev. C 72 (2005) 014906 [arXiv:hep-ph/0502248].

[18] F. M. Liu, T. Hirano, K. Werner and Y. Zhu, J. Phys. G 36 (2009) 064072 [arXiv:0811.0666 [hep-ph]].
[19] F. M. Liu, T. Hirano, K. Werner and Y. Zhu, Phys. Rev. C 80 (2009) 034905 [arXiv:0902.1303 [hep-ph]].

[20] K. Dusling, Nucl. Phys. A 839 (2010) 70 [arXiv:0903.1764 [nucl-th]].

[21] K. Dusling and I. Zahed, arXiv:0911.2426 [nucl-th].

[22] M. M. Aggarwal et al. [WA98 Collaboration], arXiv:nuclex/0006007.

[23] M. M. Aggarwal et al. [WA98 Collaboration], Phys. Rev. Lett. 85 (2000) 3595 [arXiv:nucl-ex/0006008].

[24] M. M. Aggarwal et al. [WA98 Collaboration], Phys. Rev. Lett. 93 (2004) 022301 [arXiv:nucl-ex/0310022].

[25] A. Adare et al. [PHENIX Collaboration], Phys. Rev. C 81 (2010) 034911 [arXiv:0912.0244 [nucl-ex]].

[26] S. A. Bass et al., Prog. Part. Nucl. Phys. 41 (1998) 255 [Prog. Part. Nucl. Phys. 41 (1998) 225] [arXiv:nuclth/9803035].

[27] M. Bleicher et al., J. Phys. G 25 (1999) 1859 [arXiv:hep$\mathrm{ph} / 9909407]$.

[28] H. Petersen, M. Bleicher, S. A. Bass and H. Stocker, arXiv:0805.0567 [hep-ph].

[29] D. H. Rischke, S. Bernard and J. A. Maruhn, Nucl. Phys. A 595 (1995) 346 [arXiv:nucl-th/9504018].

[30] D. H. Rischke, Y. Pursun and J. A. Maruhn, Nucl. Phys. A 595 (1995) 383 [Erratum-ibid. A 596 (1996) 717] [arXiv:nucl-th/9504021].

[31] H. Petersen, J. Steinheimer, G. Burau, M. Bleicher and H. Stocker, Phys. Rev. C 78 (2008) 044901 [arXiv:0806.1695 [nucl-th]].

[32] F. Cooper and G. Frye, Phys. Rev. D 10 (1974) 186.

[33] J. Steinheimer, V. Dexheimer, H. Petersen, M. Bleicher, S. Schramm and H. Stoecker, [arXiv:0905.3099 [hep-ph]].

[34] D. Zschiesche, S. Schramm, J. Schaffner-Bielich, H. Stoecker and W. Greiner, Phys. Lett. B 547 (2002) 7 [arXiv:nucl-th/0209022].

[35] J. Steinheimer, S. Schramm and H. Stocker, arXiv:0909.4421 [hep-ph].

[36] UrQMD v3.3 is available at http://urqmd.org/ 